PROCEEDINGS OF THE

AMERICAN MATHEMATICAL SOCIETY

Volume 135, Number 6, June 2007, Pages 1895-1904

S 0002-9939(07)08688-1

Article electronically published on January 12, 2007

\title{
MORE LIMIT CYCLES THAN EXPECTED IN LIÉNARD EQUATIONS
}

\author{
FREDDY DUMORTIER, DANIEL PANAZZOLO, AND ROBERT ROUSSARIE
}

(Communicated by Carmen C. Chicone)

\begin{abstract}
The paper deals with classical polynomial Liénard equations, i.e. planar vector fields associated to scalar second order differential equations $x^{\prime \prime}+f(x) x^{\prime}+x=0$ where $f$ is a polynomial. We prove that for a well-chosen polynomial $f$ of degree 6 , the equation exhibits 4 limit cycles. It induces that for $n \geq 3$ there exist polynomials $f$ of degree $2 n$ such that the related equations exhibit more than $n$ limit cycles. This contradicts the conjecture of Lins, de Melo and Pugh stating that for Liénard equations as above, with $f$ of degree $2 n$, the maximum number of limit cycles is $n$. The limit cycles that we found are relaxation oscillations which appear in slow-fast systems at the boundary of classical polynomial Liénard equations. More precisely we find our example inside a family of second order differential equations $\varepsilon x^{\prime \prime}+f_{\mu}(x) x^{\prime}+x=0$. Here, $f_{\mu}$ is a well-chosen family of polynomials of degree 6 with parameter $\mu \in \mathbb{R}^{4}$ and $\varepsilon$ is a small positive parameter tending to 0 . We use bifurcations from canard cycles which occur when two extrema of the critical curve of the layer equation are crossing (the layer equation corresponds to $\varepsilon=0$ ). As was proved by Dumortier and Roussarie (2005) these bifurcations are controlled by a rational integral computed along the critical curve of the layer equation, called the slow divergence integral. Our result is deduced from the study of this integral.
\end{abstract}

\section{INTRODUCTION}

Hilbert's 16th problem, or at least the second part of this problem, asks for the maximal number of limit cycles that a polynomial planar vector field (twodimensional autonomous differential equation) can have, depending on the degree of the equation. This problem was put forward in 1900 and in the meantime has not yet been solved, notwithstanding the vast amount of papers on this subject. It is not even known whether there exists an upper bound for the number of limit cycles, only depending on the degree of the polynomial vector field, not even for degree two. According to S. Smale $[\underline{S}$, it might be more appropriate to restrict the question to the classical Liénard equation associated to a scalar second-order differential equation:

$$
\ddot{x}+f(x) \dot{x}+x=0,
$$

Received by the editors June 29, 2005 and, in revised form, February 27, 2006.

2000 Mathematics Subject Classification. Primary 34C05, 34C26.

Key words and phrases. Limit cycles, Liénard equation, slow-fast equation.

(C)2007 American Mathematical Society Reverts to public domain 28 years from publication 
with $f$ a polynomial of degree $2 n, \quad n \in \mathbb{N}$. One says that the Liénard equation is classical because the conservative term in (1) is linear. To this equation one associates the following classical polynomial Liénard equation:

$$
\left\{\begin{array}{l}
\dot{x}=y-F(x) \\
\dot{y}=-x
\end{array}\right.
$$

where $F(x)=\int_{0}^{x} f(s) d s$ is a polynomial of degree $2 n+1$.

Let $\boldsymbol{L}$ be the vector field defined by the differential equation (2). For any polynomial $f$, the vector field $\boldsymbol{L}$ has a unique singular point located at the origin. Each limit cycle of this field surrounds the origin. Now, let us suppose that the polynomial $f$ is replaced by a family $f_{\alpha}$ of polynomials of degree $2 n$, with $\alpha$ near $0 \in \mathbb{R}^{p}$ and $f_{0}=0$. This family of vector fields is a perturbation of a Hamiltonian vector field with Hamiltonian $\frac{1}{2}\left(x^{2}+y^{2}\right)$, and it is easy to prove that no more than $n$ limit cycles bifurcate when the parameter $\alpha$ bifurcates from $0 \in \mathbb{R}^{p}$. This result among other ones was obtained by Lins Neto, de Melo and Pugh in LMP. This bound for the number of limit cycles in the vicinity of the simple Hamiltonian vector field led these authors to conjecture that this number is also globally valid.

Conjecture of Lins Neto, de Melo and Pugh. The number of limit cycles in a Liénard equation (2), with $F$ a polynomial of degree $2 n+1$, is at most $n$.

The conjecture, known to be correct for $n=1$, was still open for $n \geq 2$. We want to show that this conjecture has counterexamples for $n \geq 3$, starting with $n=3$.

Theorem 1.1. There exists a polynomial $f$ of degree 6 such that the associated Liénard equation (2) has at least four limit cycles.

Remark 1.2. Based on the result for $n=3$, it suffices, for $n>3$, to add to $f$ the following terms: $\varepsilon_{i} x^{2 i}$, for $i \geq 4$, with $\varepsilon_{i}$ of alternating sign and decreasing to zero sufficiently fast, when $i \rightarrow \infty$, in order to let the required number of extra limit cycles bifurcate from infinity.

Our method to prove the existence of $f$ in Theorem 1.1 is to search for the corresponding equation (2) inside a parameter family of slow-fast systems. We will make a brief review on slow-fast systems and results about them in the next paragraph. For the moment we want to concentrate on the particular example under study in this paper. First, we note that we can introduce a positive coefficient $\varepsilon$ in the front term in the second line of (2), just by making the change of variables $(x, y) \rightarrow(x, \sqrt{\varepsilon} y)$ and the change of time $t \rightarrow \frac{t}{\sqrt{\varepsilon}}$. Next, we can suppose that the singular point is not fixed at the origin but is situated at a moving position $(\lambda, F(\lambda))$, where $\lambda \in \mathbb{R}$ is a parameter. Finally, we can suppose that $F$ depends on extra parameters. More precisely, we want to consider the three-parameter family $P_{c, e}(x)+a x, \quad a \in \mathbb{R}, \quad(c, e) \in R^{2}$, where

$$
P_{c, e}(x)=\left(x^{2}-1\right)^{2}(\text { c e } x+1)\left(x^{2}+e x+1 / 8\right) .
$$

For $\varepsilon$ close to zero we will obtain the slow-fast Liénard system

$$
\boldsymbol{L}_{(c, e, \lambda, a, \varepsilon)}:\left\{\begin{array}{l}
\dot{x}=y-P_{c, e}(x)-a x, \\
\dot{y}=-\varepsilon(x-\lambda),
\end{array}\right.
$$

as a function of the parameter $(c, e, \lambda, a, \varepsilon) \in \mathbb{R}^{+} \times \mathbb{R}^{+} \times \mathbb{R} \times \mathbb{R} \times \mathbb{R}^{+}$near $(0,0,0,0,0)$. For $\varepsilon=0$, we have a family of layer equations whose critical curves (also called slow 


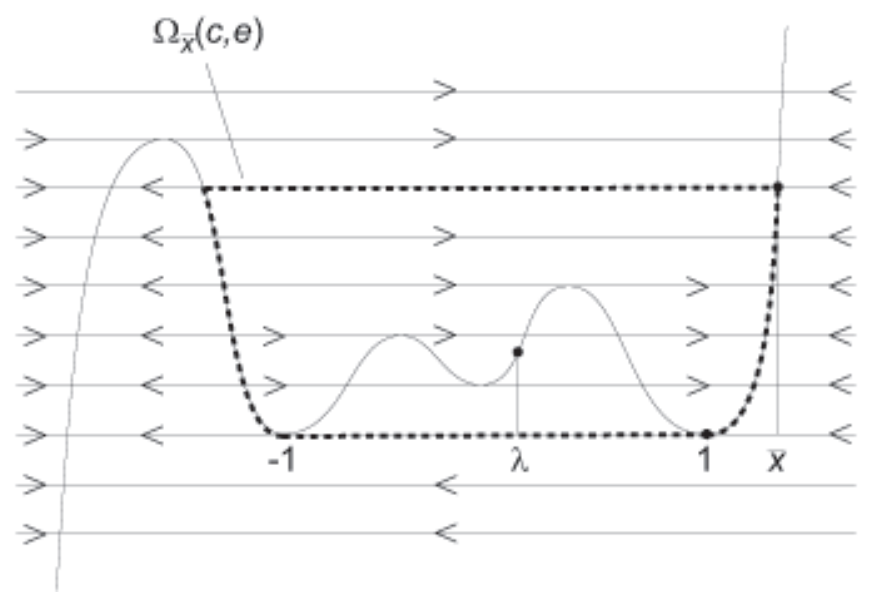

Figure 1. The layer equation for $a=0, c, e>0$.

curves) are given by $y=P_{c, e}(x)+a x$. We can interpret these limit layer equations as systems at the boundary of the space of Liénard systems $[\mathbb{R}$.

A slow-fast cycle of a layer equation is a simple closed curve, union of regular trajectories (horizontal arcs in the complement of the slow curve) and arcs on the slow curve (critical arcs). A slow-fast cycle is called common if all its critical arcs have the same type: attracting or repelling. Such a common cycle bifurcates in a single hyperbolic limit cycle. If a slow-fast cycle contains critical arcs of different type, it is called a canard cycle.

For $a \neq 0$, the layer equation contains just common cycles in number less than 3. But, for $a=0$, and for any $\bar{x}>m_{0}=1.1208006344 \ldots$ (the largest root of the equation $\left.P_{0,0}(x)=P_{0,0}\left(\frac{1}{2}\right)\right)$, the layer equation of (4) for $\varepsilon=0$ has a canard cycle $\Omega_{\bar{x}}(c, e)$ if $c>0$ and $e>0$ are small enough. This canard cycle contains a regular trajectory at the level $y=P_{c, e}(\bar{x})$ and the two minima $( \pm 1,0)$. It also contains one attracting and one repelling critical arc (see Figure 1). Theorem 1.1 is a consequence of the fact that this canard cycle $\Omega_{\bar{x}}(c, e)$ can bifurcate in multiple limit cycles. The precise result is as follows.

Theorem 1.3. Let $m_{0}$ be the constant defined above, and let $m, M$ be two real numbers such that $m_{0}<m<M$. Then, there exist two analytic functions $C(e, \bar{x}), \Lambda(e, \bar{x})$ defined for $(e, \bar{x}) \in[0, e(m, M)] \times[m, M]$, where $e(m, M)$ as a function of $(m, M)$ is small, such that if we take any fixed value $(e, \bar{x}) \in[0, e(m, M)] \times[m, M]$, the reduced system $\tilde{\boldsymbol{L}}_{(\lambda, a, \varepsilon)}=\boldsymbol{L}_{(C(e, \bar{x}), e, \lambda, a, \varepsilon)}$, depending on the parameters $(\lambda, a, \varepsilon)$, has the following properties:

(i) There exists a continuous function a $(\varepsilon)$ such that $a(0)=0$ and, for any fixed value $\varepsilon>0$ small enough, the vector field associated to $\tilde{\boldsymbol{L}}_{(\lambda, a, \varepsilon)}$ for $\lambda=\Lambda(e, \bar{x})$ and $a=a(\varepsilon)$ has a limit cycle of order three $\Gamma(\varepsilon)$. Moreover, $\Gamma(\varepsilon)$ unfolds generically in a function of the parameters $\tilde{\lambda}=\lambda-\Lambda(e, \bar{x})$ and $\tilde{a}=a-a(\varepsilon)$, producing three hyperbolic limit cycles for arbitrarily small values of $\tilde{\lambda}$ and $\tilde{a}$.

(ii) For $\varepsilon \rightarrow 0, \Gamma(\varepsilon) \rightarrow \Omega_{\bar{x}}(C(e, \bar{x}), e)$ (in the Hausdorff sense), and this canard cycle is contained in the interior of an annulus $A$ which has the following property: 
for $\varepsilon>0$ small enough, the vector field defined by $\tilde{\boldsymbol{L}}_{(\lambda, a, \varepsilon)}$ is transverse to the boundary, pointing inward along one component and outward along the other one.

Theorem 1.3 easily implies Theorem 1.1. In fact the point (i) gives values of the parameter $(c, e, \lambda, a, \varepsilon)$ for which we have three hyperbolic limit cycles contained in an annulus in which the total number of limit cycles, counted with their multiplicity must be even, as it follows from the Poincaré-Bendixson Theorem. Then, this number of limit cycles is $\geq 4$.

Remark 1.4. For a chosen $(e, \bar{x})$, the point (ii) says that three of the limits cycles tend to the canard cycle $\Omega_{\bar{x}}(c, e)$ with $c=C(e, \bar{x})$, when $\varepsilon$ tends to 0 . The other ones are also odd in number and most probably there is only one. In case a Hausdorff limit exists, for $\varepsilon \rightarrow 0$, it will be another one of the canard cycles in the 1-parameter family of intermediate canard cycles. Its shape does not need to be similar to the shape of the triple cycle.

On the other hand, as it follows from Theorem 1 of DR3], there is a constant $K>0$ such that for $(a, \lambda)$ near $(0, \Lambda(e, \bar{x}))$ and $\varepsilon \in] 0, \varepsilon_{0}\left[\right.$, the system $\tilde{\boldsymbol{L}}_{(\lambda, a, \varepsilon)}$ has just 2 (hyperbolic) limit cycles for $a<a(\varepsilon)-e^{-\frac{K}{\varepsilon}}$ and no limit cycle for $a>a(\varepsilon)+e^{-\frac{K}{\varepsilon}}$. As a consequence, four or more limit cycles can just coexist in an exponentially small tongue around the curve $a=a(\varepsilon)$, in the plane $(a, \varepsilon)$. From this observation, one can deduce that it will be very difficult to obtain them numerically (also because one has no precise information about the function $a(\varepsilon)$ ).

\section{Slow-FAST Systems}

2.1. Generalities. We will consider a general slow-fast Liénard system

$$
\boldsymbol{L}:\left\{\begin{array}{l}
\dot{x}=y-F(x), \\
\dot{y}=-\varepsilon(x-\lambda),
\end{array}\right.
$$

where $F$ is a Morse function. If $p_{i}, i=1, \cdots, k$ are the critical points of $F$, we denote by $P_{i}=\left(p_{i}, F\left(p_{i}\right)\right)$ the corresponding points on the slow curve of the layer equation. These points are ending points of critical arcs which are normally hyperbolic: attracting or repelling. Along any compact subinterval of these arcs are attached 2-dimensional central manifolds, transverse to the plane $\{\varepsilon=0\}$. These manifolds are often called slow manifolds, and their existence follows from the Fenichel Theory.

The singular point $s(\lambda)=(\lambda, F(\lambda)$ ) (which is the unique singular point of $\boldsymbol{L}$ when $\varepsilon>0$ ) separates the slow curve in two parts: on the left side, the direction induced by the motion on the slow manifold is upward, and on the right side this direction is downward. These directions of motion on the critical arcs are called directions of the slow dynamics.

We have already introduced notions about slow-fast cycles in the particular slowfast system given in the Introduction, but these notions also remain valid for (5). A necessary condition for a slow-fast cycle is that it can be oriented in a way compatible with the direction of the regular orbits, outside the slow curve, and with the direction of the slow dynamics inside the slow curve. Recall that the slow-fast cycles may be of common or of canard type. The common cycles are said to be attracting or repelling, depending on the type of their critical arcs. Perturbations from common cycles are easily understood, using a desingularization of $\boldsymbol{L}$ at the critical points of the slow curve (see [DR1]), also called contact points: 
each attracting common cycle perturbs into a unique hyperbolic attracting limit cycle, and similar for the repelling common cycle.

A generic slow-fast system $\boldsymbol{L}$ corresponds to a Morse function with distinct critical values $\left(F\left(p_{i}\right) \neq F\left(p_{j}\right)\right.$ if $\left.p_{i} \neq p_{j}\right)$ and such that $\lambda$ is not a critical point of $F$. A generic $\boldsymbol{L}$ has just slow-fast cycles of common type, and their number is no more than $n$, if $F$ is a polynomial of degree $2 n+1$. As a consequence, a generic polynomial system $\boldsymbol{L}$ of degree $2 n+1$ has no more than $n$ limit cycles if $\epsilon$ is small enough. This result seems to give more strength to the conjecture in LMP.

Canard cycles can occur, for instance, when the singular point is located at a critical point of the slow curve, or when two extrema of the same type on the slow curve have the same value and are located on each side of the singular point. In the first case one has a bifurcation of Van der Pol type which can produce multiple canard cycles giving rise to multiple limit cycles [DR2]. The second case was studied recently in DR3]. Now we want to recall some of the results of [DR3] on which is based the present paper.

We will suppose that $F$ is replaced in (5) by a polynomial family $F_{\nu, a}(x)$ with $(\nu, a) \in \mathbb{R}^{p} \times \mathbb{R}$ near $\left(\nu_{0}, 0\right)$. We call $\boldsymbol{L}_{\nu, \lambda, a, \varepsilon}$ the corresponding family of slow-fast systems. To be coherent with our example (4), we will suppose that $F_{\nu, 0}$ has two minima at the same level, that these minima are positioned at \pm 1 , and that

$$
\left.\frac{\partial\left(F_{0, a}(1)-F_{0, a}(-1)\right)}{\partial a}\right|_{a=0} \neq 0 .
$$

Let $\gamma_{l}(\nu)$ be the repelling critical arc which starts at $\left(-1, F_{\nu, 0}(-1)\right)$, and $\gamma_{r}(\nu)$ the attracting critical arc arriving at $\left(1, F_{\nu, 0}(1)\right)$ (following the direction of the slow dynamics). The $\operatorname{arc} \gamma_{r}(\nu)$ is parametrized by $\left.x \in\right] 1, p^{\prime}(\nu)\left[\right.$, where $p^{\prime}(\nu)$ is the next critical point of $F_{\nu, 0}$ on the right of 1 or is $\infty$ if there in no such critical point. We suppose that there exists an interval $[m, M] \subset] 1, p^{\prime}\left(\nu_{0}\right)[$ such that for each $x \in[m, M]$ and $\nu$ near $\nu_{0}$, the regular orbit $R(\nu, x)$ of the layer equation $\boldsymbol{L}_{\nu, \lambda, 0,0}$ arriving at $\left(x, F_{\nu, 0}(x)\right)$ starts at a point of $\gamma_{l}(\nu)$ with $x$-coordinate $\mathfrak{z}(\nu, x)$. For each $x \in[m, M]$, one has a canard cycle $\Omega_{x}(\nu)$ which contains the regular orbit at the level $\left\{y=F_{\nu, 0}(x)\right\}$. An essential tool to study the bifurcations of the canard cycle $\Omega_{x}(\nu)$ is the slow divergence integral

$$
I_{\nu, \lambda}(x)=\int_{\mathfrak{z}(\nu, x)}^{-1} \frac{F_{\nu, 0}^{\prime}(s)^{2}}{s-\lambda} d s+\int_{1}^{x} \frac{F_{\nu, 0}^{\prime}(s)^{2}}{s-\lambda} d s
$$

defined and analytic for $x \in[m, M]$ and $(\nu, \lambda)$ near $\left(\nu_{0}, 0\right)$. It denotes the integral of the divergence, calculated at the critical points of the canard cycle, and integrated over the slow time. $I_{\nu, \lambda}(x)$ represents the limit, for $\varepsilon \rightarrow 0$, of $\varepsilon \tilde{I}_{\nu, \lambda, \varepsilon}(\gamma)$, where $\tilde{I}_{\nu, \lambda, \varepsilon}(\gamma)$ stands for the integral of the divergence along limit cycles $\gamma$ of the perturbed system. Let us consider some $\bar{x} \in[m, M]$. Through the diffeomorphism $x \rightarrow y=F_{\nu, 0}(x)$ defined in a neighborhood of $\bar{x}$, one can use $x$ to parametrize a transverse section $\Sigma$ to $R(\nu, \bar{x})$. Up to the notation, the following result is a particular case of the Theorem 2 of [DR3].

Let us suppose that condition (66) is fulfilled and that for some $\nu_{0} \in \mathbb{R}^{p}$ the $\lambda$ family $I_{\nu_{0}, \lambda}(x)$ defines a generic 1 -parameter unfolding of fold type, at $\left(\bar{x}, \lambda_{0}\right)$. Then for $\varepsilon>0$ sufficiently small, there exists a continuous function $a(\varepsilon)$ with $a(0)=0$ such that the $(\lambda, a)$-family $\boldsymbol{L}_{\nu_{0}, \lambda, a, \varepsilon}$ contains a limit cycle of multiplicity 3 near $\Omega_{\bar{x}}\left(\nu_{0}\right)$ for $a=a(\varepsilon)$ and $\lambda=\lambda_{0}$. Such a limit cycle unfolds generically in function of the parameters $\tilde{\lambda}=\lambda-\lambda_{0}$ and $\tilde{a}=a-a(\varepsilon)$. 
2.2. The slow divergence integral. The result that we recalled in the previous subsection reduces the study of the limit cycles bifurcating from the canard cycle $\Omega_{\bar{x}}\left(\nu_{0}\right)$ to the study of the bifurcation of the zeros of the family of integrals $I_{\nu, \lambda}(x)=$ $I(\nu, \lambda, x)$, which we simply call the slow divergence integral. From now on we will suppose that $F_{0,0}(x)=F_{0,0}(-x)$.

First, let us observe that $\mathfrak{z}(\nu, x)$ is an analytic function defined for $\nu$ near 0 by the implicit relation

$$
F_{\nu, 0}(\mathfrak{z}(\nu, x))=F_{\nu, 0}(x) \quad \text { and } \quad \mathfrak{z}(0, x)=-x .
$$

Let us compute some partial derivatives of $I$. First of all, using the fact that $F^{\prime}(0, x)^{2} / x^{2}$ is an even function, we get

$$
\left.\frac{\partial I}{\partial \lambda}(\nu, \lambda, x)\right|_{(\nu, \lambda)=(0,0)}=\int_{-x}^{-1} \frac{F_{0,0}^{\prime}(s)^{2}}{s^{2}} d s+\int_{1}^{x} \frac{F_{0,0}^{\prime}(s)^{2}}{s^{2}} d s=2 \int_{1}^{x} \frac{F_{0,0}^{\prime}(s)^{2}}{s^{2}} d s>0
$$

for all $x>1$.

To compute the partial derivatives with respect to $x$, let us observe that

$$
\frac{\partial \mathfrak{z}}{\partial x}(\nu, x)=\frac{F_{\nu, 0}^{\prime}(x)}{F_{\nu, 0}^{\prime}(\mathfrak{z}(e, x))}
$$

and therefore,

$$
\frac{\partial I}{\partial x}(\nu, \lambda, x)=F_{\nu, 0}^{\prime}(x)\left(\frac{F_{\nu, 0}^{\prime}(x)}{x-\lambda}-\frac{F_{\nu, 0}^{\prime}(\mathfrak{z}(\nu, x))}{\mathfrak{z}(\nu, x)-\lambda}\right) .
$$

Therefore, the function

$$
\lambda(\nu, x)=\frac{F_{\nu, 0}^{\prime}(\mathfrak{z}(\nu, x)) x-F_{\nu, 0}^{\prime}(x) \mathfrak{z}(\nu, x)}{F_{\nu, 0}^{\prime}(\mathfrak{z}(\nu, x))-F_{\nu, 0}^{\prime}(x)}
$$

is such that the relation $\frac{\partial I}{\partial x}(\nu, \lambda(\nu, x), x)=0$ holds. Differentiating both sides of this relation with respect to $x$, we get

$$
\frac{\partial^{2} I}{\partial x^{2}}(\nu, \lambda(\nu, x), x)=-\frac{\partial^{2} I}{\partial x \partial \lambda}(\nu, \lambda(\nu, x), x) \frac{\partial \lambda}{\partial x}(\nu, x) .
$$

Note that $\frac{\partial^{2} I}{\partial x \partial \lambda}(\nu, \lambda, x)$ is given by

$$
\frac{\partial^{2} I}{\partial x \partial \lambda}(\nu, \lambda, x)=F_{\nu, 0}^{\prime}(x)\left(\frac{F_{\nu, 0}^{\prime}(x)}{(x-\lambda)^{2}}-\frac{F_{\nu, 0}^{\prime}(\mathfrak{z})}{(\mathfrak{z}-\lambda)^{2}}\right)
$$

and therefore $\frac{\partial^{2} I}{\partial x \partial \lambda}(0,0, x)=2\left(\frac{F_{0,0}^{\prime}(x)}{x}\right)^{2}>0$ for $x>1$ (we use the assumption that $F_{0,0}(x)$ is even).

In particular, we have the following relation (for $(\nu, \lambda)$ sufficiently near $(0,0)$ ):

$$
\frac{\partial^{2} I}{\partial x^{2}}(\nu, \lambda(\nu, x), x)=0 \quad \text { if and only if } \quad \frac{\partial \lambda}{\partial x}(\nu, x)=0 .
$$

\section{Four himit CyCles AT DEGReE SeVen}

Now, consider a family $F_{\nu, a}(x)$ with $\nu=(c, e) \in \mathbb{R}^{2}$ equal to $P(c, e, x)+a x$, where $P(c, e)$ is the 2-parameter family of polynomials

$$
P(c, e, x)=\left(x^{2}-1\right)^{2}(\text { ce } x+1)\left(x^{2}+e x+1 / 8\right) .
$$


Let us observe that $F_{\nu, a}$ is of degree 7 with a positive leading coefficient as soon as $c \in \mathbb{R}^{+}$and $e \in \mathbb{R}^{+}$. Let us also observe that condition (6) is trivially verified. Then, we just have to consider $F_{\nu, 0}(x)=P(c, e, x)$. For $e=0$, the polynomial degenerates to a symmetric Morse polynomial of degree 6 , with 2 minima located at \pm 1 , having common value 0 , two maxima at $\pm \frac{1}{2}$, having a common value $\frac{27}{128}$, and another minimum at 0 . Due to the symmetry, the canard cycles passing through the points $( \pm 1,0)$ have a related slow divergence integral identically equal to zero. These canard cycles $\Omega_{x}$ are defined for $\left.\left.x \in\right] m_{0},+\infty\right)$, where $m_{0}$ is the biggest root of the equation $P(0,0, x)=P\left(0,0, \pm \frac{1}{2}\right)$.

We want to study the perturbation of this situation when $e, c$ are positive and $e$ is sufficiently small. For such a perturbation, all the critical points are preserved. Two minima at \pm 1 are fixed, keeping the same value 0 ; the maxima at $\pm \frac{1}{2}$ are slightly pushed in position $p_{1}(c, e)=-\frac{1}{2}+O(e)$ and $p_{2}(c, e)=\frac{1}{2}+O(e)$. We call them the internal maxima. Between these two points, we have a minimum near the origin.

The following two lemmas show that for $e>0$ small enough, the slow divergence integral $I(c, e, \lambda, x)$ related to canard cycles through $( \pm 1,0)$ has generic $\lambda$-parameter bifurcations at any point $x$, for some value $c=C(e, x)$ and at some value $\Lambda(e, x)$ of $\lambda$.

Lemma 3.1. Choose any $m, M$ with $m_{0}<m<M$. There exist two analytic functions $C(e, x), \Lambda(e, x)$ for $(e, x) \in[0, e(m, M)] \times[m, M]$, where $e(m, M)>0$ is small, in function of $(m, M)$. Moreover $C(e, x)$ is strictly positive for all sufficiently small $e>0$ and $\Lambda(0, x)=0$.

The slow divergence integral $I(c, e, x)$ is defined in a neighborhood of $\{0\} \times\{0\} \times$ $[m, M]$, and the functions $C, \Lambda$ satisfy the following equations:

$$
I(C(e, x), e, \Lambda(e, x), x)=0 \quad \text { and } \quad \frac{\partial I}{\partial x}(C(e, x), e, \Lambda(e, x), x)=0 .
$$

Moreover, one can choose e $(m, M)$ such that

$$
\frac{\partial^{2} I}{\partial x^{2}}(C(e, x), e, \Lambda(e, x), x)>0
$$

for all $(e, x) \in] 0, e(m, M)] \times[m, M]$ (i.e. for $e>0)$.

Proof. Formally replacing the series $\mathfrak{z}(c, e, x)=-x+\alpha_{1}(c, x) e+O\left(e^{2}\right)$ into the expression (8) we get

$$
\mathfrak{z}(c, e, x)=-x-\frac{1}{6} \frac{\left(x^{2}-1\right)\left(8 c x^{2}+c+8\right)}{4 x^{2}-1} e+O\left(e^{2}\right) .
$$

Replacing $\mathfrak{z}(c, e, x)$ by the above series expansion in (11), we get a result that we denote by $\lambda_{1}(c, e, x)=Q(c, x) e+O\left(e^{2}\right)$, where $Q(c, x)$ is the function

$$
Q(c, x)=-\frac{1}{12} \frac{96 c x^{6}+32 x^{4}-68 c x^{4}+25 c x^{2}+8 x^{2}+8+c}{\left(4 x^{2}-1\right)^{2}} .
$$

On the other hand, replacing the series of $\mathfrak{z}(c, e, x)$ into the equation

$$
I(c, e, \lambda, x)=0
$$


and solving for $\lambda$, we get a result that we denote by $\lambda_{2}(c, e, x)=S(c, x) e+O\left(e^{2}\right)$, where $S(c, x)=S_{1}(c, x) / S_{2}(x)$, with

$$
\begin{aligned}
S_{1}(c, x)= & -\frac{1}{132}\left(30240 c x^{8}+90720 x^{7} c+12320 x^{6}+81340 c x^{6}+2100 x^{5} c\right. \\
& +36960 x^{5}-19785 c x^{4}+46200 x^{4}+40040 x^{3}+15685 x^{3} c+35112 x^{2} \\
& \left.+27429 c x^{2}+15447 c x+31416 x+6304 c+19712\right), \\
S_{2}(x)= & 560 x^{6}+1680 x^{5}+1560 x^{4}+200 x^{3}-321 x^{2}-3 x+104 .
\end{aligned}
$$

Let us note that the polynomial $S_{2}(x)$ has no real root. To obtain a function $C(e, x)$ which satisfies our requirements, we have to solve the equation

$$
\lambda_{2}(c, e, x)-\lambda_{1}(c, e, x)=e(S(c, x)-Q(c, x))+e^{2} \Psi(c, e, x)=0,
$$

where $\Psi$ is an analytic function. This is equivalent to solving:

$$
S(c, x)-Q(c, x)+e \Psi(c, e, x)=0 .
$$

Now $S(c, x)-Q(c, x)=\frac{c_{2}(x) c-c_{1}(x)}{S_{2}(x)\left(-1+4 x^{2}\right)^{2}}$ with

$$
\begin{aligned}
c_{1}(x)= & 88\left(80 x^{7}+320 x^{6}+568 x^{5}+672 x^{4}+549 x^{3}+116 x^{2}-80 x-20\right), \\
c_{2}(x)= & 17920 x^{11}+71680 x^{10}+99840 x^{9}+40960 x^{8}-28176 x^{7}-30784 x^{6} \\
& -13016 x^{5}-21024 x^{4}-22783 x^{3}-4572 x^{2}+3440 x+860 .
\end{aligned}
$$

As the polynomials $c_{1}$ and $c_{2}$ have no real root greater than or equal to 1 , one can define $c(x)=c_{1}(x) / c_{2}(x)$ for $x \geq 1$, and this function is strictly positive. The equation (14) is then equivalent to an equation $c-c(x)-e \tilde{\Psi}(c, e, x)=0$, which can be solved using the Implicit Function Theorem to give the required function $c=C(e, x)>0$, for $(e, x) \in[0, e(m, M)] \times[m, M]$, where $m_{0}<m<M$ and $e(m, M)>0$ is small, in function of $(m, M)$. We can now define $\Lambda(e, x)=$ $\lambda(C(e, x), e, x)$. To conclude the proof, we verify that $\frac{\partial^{2} I}{\partial x^{2}}(C(e, x), e, \Lambda(e, x), x)>0$ for all sufficiently small $e>0$. Using the relation (12), it suffices to see that the function $\frac{\partial \Lambda}{\partial x}(e, x)=R(x) e+O\left(e^{2}\right)$ is such that $R(x)>0$ for all $x>1$. The function $R(x)$ can be explicitly obtained by replacing the function $c=c(x)$ into the expression of $Q(c, x)$ (or $S(c, x)$ ) and differentiating the resulting expression with respect to $x$. Doing so, we get $R(x)=R_{1}(x) / R_{2}(x)$, where

$$
\begin{aligned}
R_{1}(x)= & 64 x(2 x+1)(2 x-1)\left(30240 x^{8}-19785 x^{4}+2100 x^{5}+90720 x^{7}\right. \\
& \left.+27429 x^{2}+15685 x^{3}+15447 x+6304+81340 x^{6}\right) \\
& \left(80 x^{8}+400 x^{7}+952 x^{6}+1560 x^{5}+1825 x^{4}+1125 x^{3}\right. \\
+ & \left.265 x^{2}+25 x+5\right), \\
R_{2}(x)= & 3\left(17920 x^{11}+71680 x^{10}+99840 x^{9}+40960 x^{8}-28176 x^{7}-30784 x^{6}\right. \\
& \left.-13016 x^{5}-21024 x^{4}-22783 x^{3}-4572 x^{2}+3440 x+860\right)^{2} .
\end{aligned}
$$

Using these expressions, we can verify that $R_{1}(x), R_{2}(x)>0$ for all $x>1$.

Let us prove that the variation of the parameter $\lambda$ in a neighborhood of $\lambda=$ $\Lambda(e, x)$ yields a generic bifurcation of $I(c, e, \lambda, x)$ (for all small $e>0$ ).

Lemma 3.2. Let us fix $\bar{x}>1$ and consider the function

$$
J(e, \tilde{\lambda}, \tilde{x})=I(C(e, \bar{x}), e, \Lambda(e, \bar{x})+\tilde{\lambda}, \bar{x}+\tilde{x}),
$$

where $\Lambda(e, \bar{x})$ and $C(e, \bar{x})$ are the functions defined in Lemma 3.1. Then, up to a reparametrization and a division by a nonzero function, the $\tilde{\lambda}$-family $J(e, \tilde{\lambda}, \tilde{x})$ is 
equal to $\tilde{\lambda}+\tilde{x}^{2}$, for each sufficiently small fixed $e>0$. This means that this family yields a generic fold bifurcation of the double zero of $J(e, 0,0)$.

Proof. Write the Taylor series expansion of $F$ in terms of $\lambda$ as

$$
J(e, \tilde{\lambda}, \tilde{x})=J_{0}(e, \tilde{x})+J_{1}(e, \tilde{x}) \tilde{\lambda}+O\left(\tilde{\lambda}^{2}\right) .
$$

Then, it follows from Lemma 3.1 that

$$
J_{0}(e, \tilde{x})=I(e, \Lambda(e, \bar{x}), C(e, \bar{x}), \bar{x}+\tilde{x})=\tilde{x}^{2} U
$$

for some function $U$ which is strictly positive for all sufficiently small $\tilde{x}$ and all sufficiently small $e>0$. We remark that $U$ vanishes identically for $e=0$.

On the other hand, it follows from (9) and the chain rule that

$$
J_{1}(0,0)=\frac{\partial I}{\partial \lambda}(0,0,0, \bar{x})>0 .
$$

Therefore, $J_{1}(e, \tilde{x})=d+O(e, \tilde{x})$, for some positive constant $d>0$.

Hence, up to a reparametrization and a division by a nonzero function, the family

$$
J(e, \tilde{\lambda}, \tilde{x})=\tilde{x}^{2} U+(d+O(e, \tilde{x})) \tilde{\lambda}+O\left(\tilde{\lambda}^{2}\right)
$$

yields a generic fold bifurcation of the double zero of $J(e, 0,0)$ (for all sufficiently small $e>0$ ).

Let us choose some $[m, M]$ with $M>m>m_{0}$ and some $\bar{x} \in[m, M]$. If $e>0$ is chosen small enough as in Lemma 3.1 we know that there exist values $c=C(e, \bar{x})>0$ and $\lambda=\Lambda(e, \bar{x})$ such that the slow divergence integral along the canard cycle $\Omega_{\bar{x}}$ undergoes a generic fold type bifurcation. Clearly, we can choose $e>0$ small enough such that $p_{1}(C(e, \bar{x}), e)<\Lambda(e, \bar{x})<p_{2}(C(e, \bar{x}), e)$.

Fixing $e>0$ and $c=C(e, \bar{x})>0$ as above, we can now finish the proof of Theorem 1.3. We take $\varepsilon>0$ small enough to apply the result of [DR3] recalled at the end of subsection 2.1: there exist values of $a$ near zero and of $\lambda$ near $\Lambda(e, \bar{x})$ such that the system $\boldsymbol{L}_{(c, e, \lambda, a, \varepsilon)}$ has a limit cycle of order three near $\Omega_{\bar{x}}$, which generically bifurcates in function of the parameter $(\lambda, a)$. Now, as $c e>0$, the circle at infinity is repelling (see $\left[\mathrm{LMP}\right.$ ): one can find a circle $\Gamma_{1}$ in $\mathbb{R}^{2}$, as large as we want, along which the vector field $\boldsymbol{L}_{(c, e, \lambda, a, \varepsilon)}$ is transversely entering.

On the other side, writing the expansion $C(e, \bar{x})=c(\bar{x})+O(e)$ it is direct to compute that

$$
P\left(c, e, p_{2}(c, e)\right)-\left.P\left(c, e, p_{1}(c, e)\right)\right|_{c=C(e, \bar{x})}=\left(\frac{9}{16}+\frac{27}{128} c(\bar{x})\right) e+O\left(e^{2}\right) .
$$

Since $c(\bar{x})>0$, we conclude that for all sufficiently small $e>0$, the value of the polynomial $P$ at the right internal maximum is strictly greater than its value at the left one.

As $p_{1}(C(e, \bar{x}), e)<\Lambda(e, \bar{x})<p_{2}(C(e, \bar{x}), e)$, this means that we can also find a circle $\Gamma_{2}$, near the invariant cycle of the layer equation passing through the critical point $\left(p_{1}, F_{\nu}\left(p_{1}\right)\right)$, along which the vector field is transversally pointing inward. Let $A$ be the annulus domain bounded by $\Gamma_{1} \cup \Gamma_{2}$. It clearly contains the canard cycle $\Omega_{\bar{x}}$ in its interior. This concludes the proof of Theorem 1.3 .

Let us briefly recall how to deduce Theorem 1.1 if $\varepsilon$ is small enough, there will exist parameter values at which the annulus $A$ contains three hyperbolic limit cycles near $\Omega_{\bar{x}}$. We can apply the Poincaré-Bendixson Theorem to the annulus $A$ : the total number of limit cycles in $A$, counted with their multiplicity, must be even. As this number is at least three, it must be greater than four. 
Remark 3.3. In degree 5, a general form (up to translations and rescaling) for a polynomial with two minima at the same value is $P(e, x)=\left(x^{2}-1\right)^{2}(e x+1)$, depending on a parameter $e \in \mathbb{R}$. It can be proven that, at least for small values of $e$, the corresponding slow-fast Liénard system has no more than two limit cycles, which is the number predicted by the conjecture of Lins Neto, de Melo and Pugh.

\section{REFERENCES}

[DR1] F. Dumortier, R. Roussarie, Canard Cycles and Center Manifolds, Memoirs of Amer. Math. Soc. Vol. 121, n 577, 1-100 (1996). MR1327208 (96k:34113)

[DR2] F. Dumortier, R. Roussarie, Multiple Canard Cycles in Generalized Liénard Equations, Journ. Diff. Equa. vol. 174, 1-29 (2001). MR.1844521 (2002k:34076)

[DR3] F. Dumortier, R. Roussarie, Bifurcation of relaxation oscillations in dimension 2, preprint I.M.B. (2005).

[LMP] A. Lins Neto, W. de Melo, C.C. Pugh, On Liénard Equations, Proc. Symp. Geom. and Topol., Springer Lectures Notes in Math. n 597, 335-357 (1977). MR0448423 (56:6730)

[R] R. Roussarie, Putting a boundary to the space of Liénard equations, to appear in Discr. and Cont. Dyn. Sys. (2005).

[S] S. Smale, Mathematical Problems for the Next Century, Springer-Verlag, New York, Vol. 20, n 2, 7-15 (1998). MR.1631413 (99h:01033)

Universiteit Hasselt, Campus Diepenbeek, Agoralaan - Gebouw D, B-3590 Diepenbeek, BELGIUM

E-mail address: freddy.dumortier@uhasselt.be

Instituto de Matemática e Estatística, Universidade de São Paulo, Rua do Matão, 1010 - SÃo PaUlo, SP, 05508-090, BraZiL

E-mail address: dpanazzo@ime.usp.br

Institut de Mathématique De Bourgogne, U.M.R. 5584 du C.N.R.S., Université de Bourgogne, B.P. 47 870, 21078 Dijon Cedex, France

E-mail address: roussari@u-bourgogne.fr 